\title{
Percutaneous nephrostomy in the management of malignant ureteral obstruction: an alternative using central venous catheter and "zero irradiation"
}

\author{
Juan C Astigueta ${ }^{1,2,3 *}$, Juan E Silva1, Benjamin A Leiva', Miracles A Abad Licham ${ }^{3}$ and Reynaldo D Kantt ${ }^{4}$ \\ ${ }^{1}$ Oncology Urology Service, Regional Institute of Neoplastic Diseases, South America \\ ${ }^{2}$ School of Medicine, Private University Antenor Orrego, South America \\ ${ }^{3}$ Urology unit, Scientia Clinical and Epidemiological Research Institute, South America \\ ${ }^{4}$ Latin American School of Interventional Ultrasound and Percutaneous Surgery Ecoasistida, Necochea, South America
}

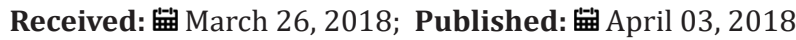

*Corresponding author: Juan C Astigueta, Oncology Urology Service Diseases, Private University Antenor Orrego, Scientia Clinical and Epidemiological Research Institute, Trujillo, Peru, South America

\section{Abstract}

Introduction and Objective: Percutaneous Nephrostomy (NP) is a procedure that involves the placement of a drainage catheter in the renal pelvis by puncture through the skin guided by an imaging method. The objective of the work is to present our experience in PN with ultrasound guidance (zero irradiation) in patients with ureteral obstruction secondary to oncological pathologies, showing the step-by-step technique and the usefulness of the 7 French central venous catheter set (CVC-7F).

Material and methods: Prospective work carried out in two institutions between 2010 and 2016. The eligibility criteria to perform percutaneous nephrostomy with ultrasound guidance (NPGE) with CVC-7F were: adult patients with oncological pathology with a dilated collecting system (moderate-severe hydronephrosis) and distance between the skin and renal pelvis less than the length of the needle. Patients with non-correctable coagulation disorder and terminal disease with ECOG 3-4 were excluded. Data on indications, technique and complications were collected.

Results: 212 NPGE were performed with CVC-7F in 189 patients with hydronephrosis secondary to oncological pathologies, 180 female and 09 male; 174 cases with diagnoses of cervical cancer, 04 of prostate, 03 of rectum, 06 of bladder, 01 of uterine sarcoma and 01 pesudotumor (genitourinary tuberculosis). The patients presented moderate-severe hydronephrosis. The main symptoms were oligo-anuria, hyperazoemia and low back pain. The average age was 59 years (range 18-83). It was performed with local anesthesia after coagulation study and consent signature. The average time of the procedure was 11 minutes and no case presented greater complication.

Conclusions: The NPGE using the set of CVC-7F, is an effective alternative of low cost, safe, reproducible and without exposure to radiation, in patients who present moderate-severe hydronephrosis and with distance between skin and dilated renal pelvis, less than the length of the needle used. We consider that the adequate choice of the patient has allowed us to have good results without major complications.

Keywords: Percutaneous ephrostomy; Ultrasound; Obstructive uropathy; Cancer

Abbreviations: NP: Percutaneous Nephrostomy; NPGE: Percutaneous Nephrostomy with Ultrasound Guidance; CVC-7F: 7 French Central Venous Catheter; ECOG: Eastern Cooperative Oncology Group 


\section{Introduction}

Table 1: Equipment and basic materials.

\begin{tabular}{|c|}
\hline Echograph with convex transducer \\
\hline 7 French central venous catheter set \\
\hline Lidocaine $2 \%$ \\
\hline Nylon $3 / 0$ \\
\hline Double Foley Probe 14 French \\
\hline Urine collection bag \\
\hline Materials of asepsis, antisepsis and sterile gloves \\
\hline Suture equipment \\
\hline Marker for skin \\
\hline Rule \\
\hline
\end{tabular}

Percutaneous Nephrostomy (NP) is a procedure that involves the placement of a drainage catheter inside the renal pelvis, through puncture through the skin guided by some imaging method such as fluoroscopy, computed tomography, ultrasound or combination of these; In general, NP is performed with fluoroscopic help and using a set of nephrostomy [1]. The technique has evolved over time, presenting different variants that have fundamentally converted it into a therapeutic procedure, which is carried out routinely in health centres that have the support of equipment, materials and trained personnel; This method, although of low morbidity, is not exempt from major complications that can compromise the patient's life, such as vessel injuries, viscera and severe infections [2]. The evolution of interventional radiology, added to the lack of training and delurologist practice, has made percutaneous nephrostomy to be carried out mainly by radiologists [3]. In our environment, as well as in countries with similar public healthcare systems, other important factors prevent routine performance of this procedure are: a) the lack of access to equipment such as the fluoroscope or tomograph that are only found in reference centers; and b) the availability and high cost of the nephrostomy set that makes it inaccessible to the vast majority of the population $[1,4]$. We present our experience in the NPGE technique using the CVC-7F set, with patients who presented obstructive uropathy at the beginning or during the evolution of their oncological disease Table 1.

\section{Material and Methods}

Descriptive work in which the data of patients with oncological pathology submitted to NPGE with CVC-7F was collected between January 2010 and December 2016

\section{Inclusion Criteria}

a. Oncological patients with moderate-severe hydronephrosis.

b. Distance of skin to dilated renal pelvis less than the length of the needle of the CVC set used (depending on the brand measures between 6.35 and $7 \mathrm{~cm}$ ).

\section{Exclusion Criteria}

a. Non-correctable coagulation disorder.

b. Terminal disease with ECOG Performance Status (scale of functional status) 3 or 4 .

\section{Preparation}

In all patients, prior to the procedure, the degree of hydronephrosis, the diameter of the renal parenchyma and the distance of the skin to the dilated collecting system were evaluated sonographically. With the informed consent, the procedure was explained to the patient, including the benefits and possible complications. Coagulation studies were performed and prophylaxis with quinolones was indicated in the majority of patients.

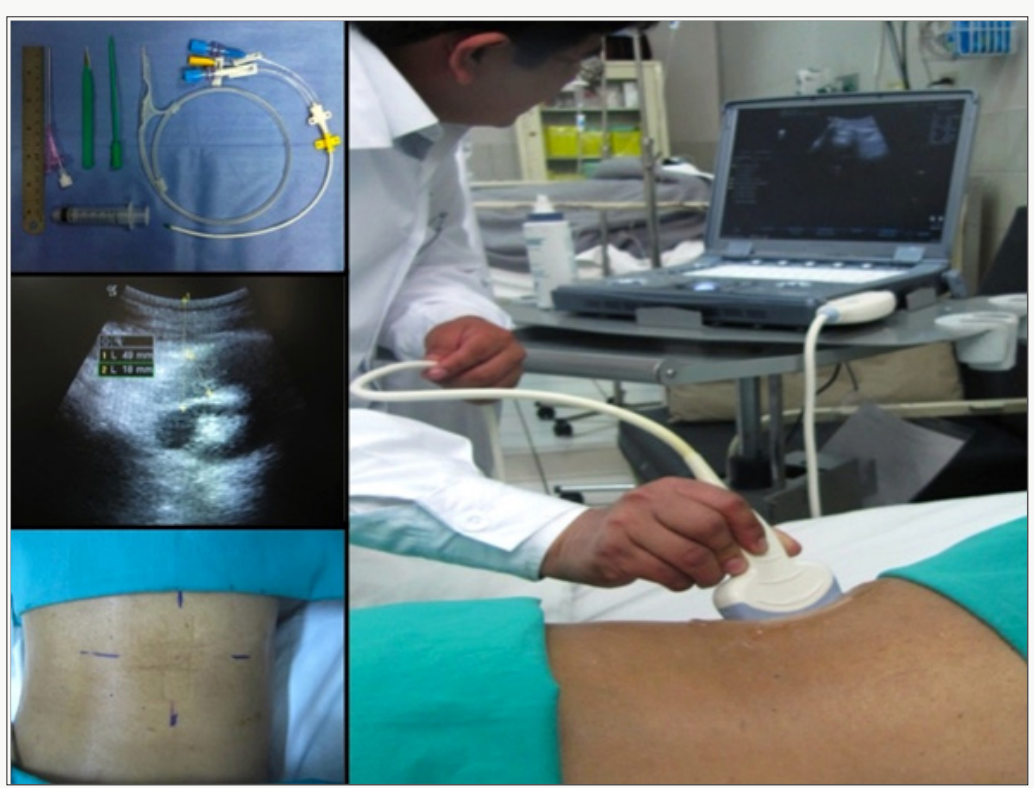

Figure 1. 


\section{Equipment and materials}

To perform the procedure we used an ultrasound scanner with its convex transducer, a set of CVC-7F that includes catheter, needle, wire guide, dilator, scalpel, syringe and other materials listed in Table 1 . The characteristics of the needle vary according to the mark, the length of those used in the present work was 6.35 to $7 \mathrm{~cm}$ and another important feature was the presence of a single or double channel in Y (Unilene $®$ ) that allowed access to the metal guide without the need to remove the syringe.

Technique:

a. Patient's position: The patient was placed in the ventral decubitus position with the upper limbs extended cephalad, in some cases it was necessary to place it in order to find the best access point.

b. Ultrasound evaluation and measurement of anatomical parameters: With the patient positioned, the distance from the skin to the dilated renal pelvis was re-evaluated, the access point was marked and the entrance angle spatially planned (Figure 1).

c. Asepsis, antisepsis and placement of sterile fields.

d. Infiltration with local anaesthetic: The skin and soft tissues were infiltrated with $2 \%$ lidocaine without epinephrine at the access point of the CVC needle.

e. Incision of the skin with scalpel at the access point: In order to decrease the resistance to access of the dilator and passage of the drainage catheter.

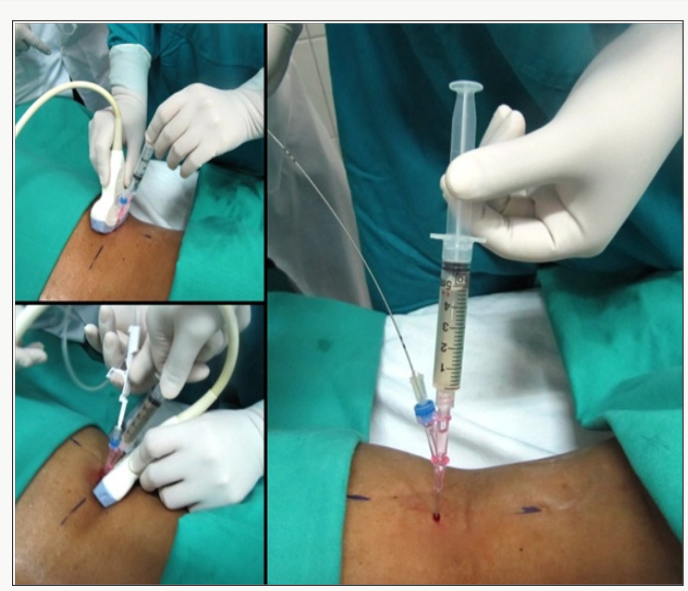

Figure 2.

f. Catheterization with Seldinger technique: Once the necessary length to enter the dilated collecting system is determined, with ultrasound guidance, the needle attached to the syringe is inserted, continuously aspirating, observing and controlling in real time the advance and access to the renal pelvis until obtaining urine. The needle is fixed and the guide is inserted. Then, the needle is removed, maintaining the guide, dilated and finally the drainage catheter is placed and the guide is removed (Figures $2 \& 3$ ).

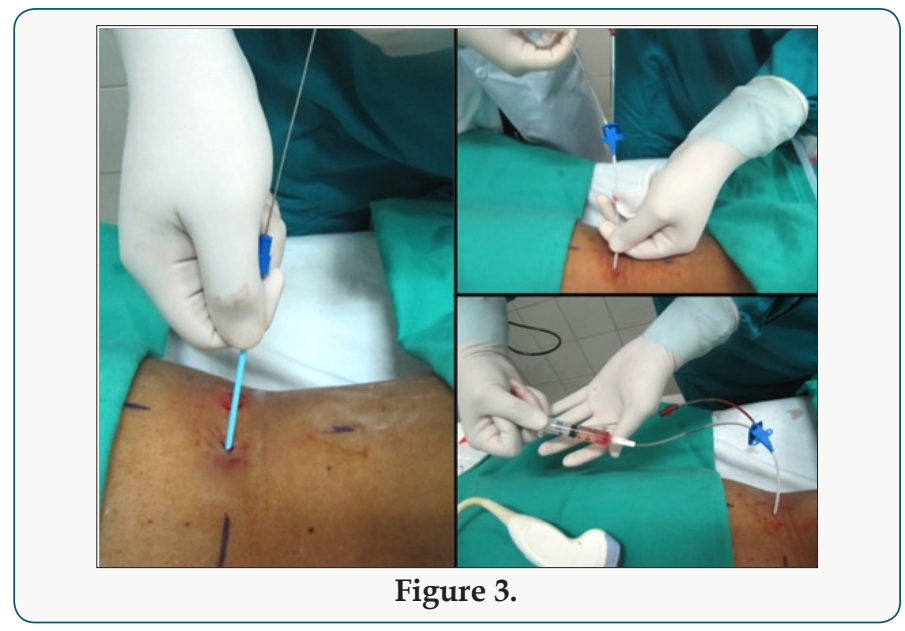

g. Catheter fixation and distal segment assembly of Foley catheter: Assessed the permeability and functionality of the catheter, it was fixed with nylon $3 / 0$ on the skin and cut the catheter at the division level in the lumens. Then, the Foley catheter was cut at the distal end and joined / adapted to the catheter. Finally it was attached to the urine collection bag (Figure 4).

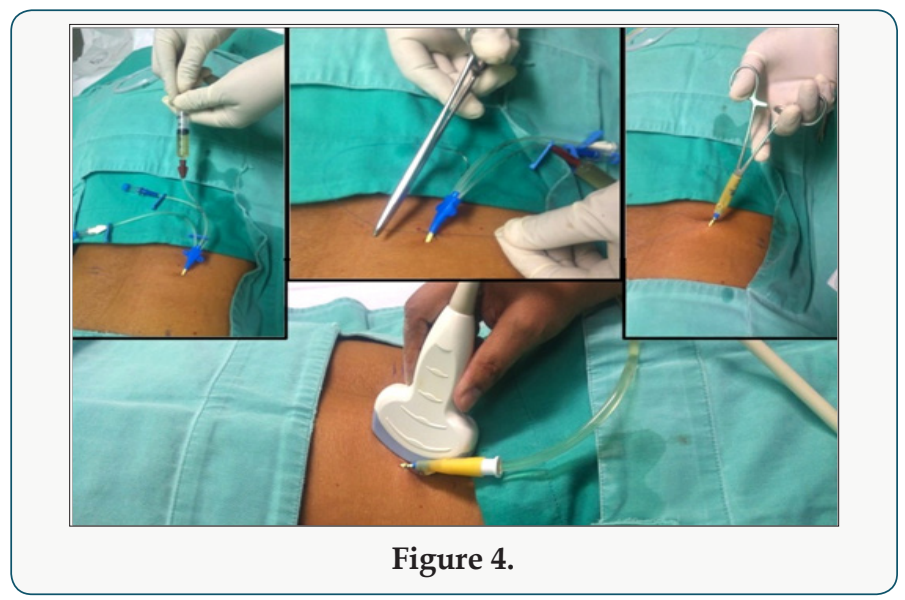

h. Patient management after the procedure: In all patients it was indicated during the initial 24 hours:

i. Monitoring of vital signs.

ii. Diuresis control every hour.

iii. Control of urine characteristics, mainly hematuria.

It was indicated, as appropriate, change of catheter the 6 weeks.

\section{Results}

212 NPGE were performed with CVC-7F in 189 patients with oncological diagnosis, 180 female and 09 male; with diagnoses of cervical cancer in 174 cases, prostate cancer in 04 cases, rectal cancer in 03 cases, bladder cancer in 06 cases, uterine sarcoma in 01 case and pseudo tumor (genitourinary tuberculosis) in 01 case. 
In the patients who presented moderate-severe hydronephrosis, the main symptoms were oligo-anuria, hyperazoemia and low back pain, and less frequently hematuria and anasarca. In cases of pure unilateral hydronephrosis (07 cases) the nephrostomy was placed to preserve the renal unit to propose the final treatment according to the primary pathology and staging. The average age was 59 years (range 18-83). The average time of the procedure was 11 minutes, of which approximately $50 \%$ was used in the ultrasound planning. $21.7 \%$ (46 cases) needed more than one attempt; none presented a major complication, in 3 patients after initially observing urine drainage, there was no production, so the catheter was permeabilized and when no diuresis was again present, it was withdrawn. The most common complications were mild transient hematuria, local pain, and involuntary catheter removal. There were no lesions of hollow or solid viscera, pneumothorax or hemorrhages that caused severe anaemia.

\section{Discussion}

The derivation of the urinary tract is the standard treatment in high obstructive uropathy, it is performed by placing endoluminal devices (ureteral catheters), percutaneous drains or canalization by conventional surgery. Nephrostomy represents one of the most effective and effective methods to prolong and improve the quality of life, there are several techniques, most of them traditionally performed under fluoroscopic control 1-3. The most common indication for nephrostomy is supravesical obstructive uropathy secondary to lithiasis, clots, post-surgical fibrosis and cancer, among the most frequent $[1,2]$. Among the primary malignancies that are mostly related to partial or complete obstruction of the urinary tract (malignant ureteral obstruction), there are those of the cervix, rectum, prostate and bladder, mainly due to tumor infiltration or extrinsic compression [5]; Other indications are urinary fistulas, hemorrhagic cystitis, access to the urinary tract for stentureteral placement and percutaneous removal of stones [6].

In 1865 Thomas Hillier in the United Kingdom reported what is probably the first publication of NP for drainage of hydronephrosis in a 4-year-old boy [7], and it was not until 1955 that Goodwin et al. [8] Described their experience and the utility of NN in the management of hydronephrosis8 since that date, the technique has evolved, being routinely used as a minimally invasive therapeutic procedure, and which is traditionally performed with radiological help 1-3. The ultrasound guidance was not published until 1974 when Jan Pedersen reported the first NPGE y and in 1976 published a series of 24 procedures in 19 patients $[9,10]$. In our country, nephrostomy is carried out mainly in reference health centres, hospitals where they have the necessary technology and materials. Seven years ago in our locality there was no suitable centre for this purpose and we did not have the equipment or materials, so we necessarily started our experience in the placement of nephrostomies using ultrasound equipment as a guide.

In the absence of the nephrostomy set that was not accessible to the patient due to its cost or availability in the environment, we used the materials from the CVC-7F set considering its similar characteristics to the original nephrostomy catheter4 (Table 2). The literature describes placement techniques such as Seldinger and direct puncture (trocar), both with specific materials for this purpose and guided by the use of imaging equipment such as fluoroscope, ultrasound or tomograph, the success rate is similar with the two techniques and varies between 92 and 94\% [1,11]. In general, the diameter of the catheter depends on the purpose of the nephrostomy and the age of the patient; a catheter of $6-10 \mathrm{~F}$ is recommended and if it is only urine, a catheter of $5-8 \mathrm{~F}$ can be used. If the collecting system is punctured for additional procedures (for example, stone removal) or in procedures complicated by macroscopic hematuria, a higher lumen catheter (14-22F) may be considered. Smaller catheters are less traumatic and easier to insert, but do not drain or decompress as effectively as larger ones $[11,12]$.

Table 2.

\begin{tabular}{|c|c|c|}
\multicolumn{1}{|c|}{} & \multicolumn{1}{|c|}{$\begin{array}{c}\text { Percutaneous } \\
\text { Nephrostomy } \\
\text { Catheter Set }\end{array}$} & $\begin{array}{c}\text { 7 French Central } \\
\text { Venous Catheter Set }\end{array}$ \\
\hline Catheter material & Polyurethane & Polyurethane \\
\hline Radiopaco & Si & And \\
\hline Catheter tip & Atraumática & Atraumática \\
\hline Catheter holes & Multiple & 2 or 3 holes \\
\hline Catheter length & 25 cm. aprox. & $20 \mathrm{~cm}$. \\
\hline Puncture needle & Longitud variable & 6.35 a 7cm. \\
\hline Cost & 250 dollars approx. & 30 dollars approx. \\
\hline
\end{tabular}

In our case, we use the materials of the CVC-7F set that has a needle for puncture of $6.35-7 \mathrm{~cm}$. (depending on the brand), a metal guide, a dilator and the drainage catheter. As referred in the methodology, the use of this material is only useful when the distance of the skin to the dilated renal pelvis is less than the length of the needle and considering the lumen, it fulfils the function of draining the urine, even something dense and with hematuria. In the literature review we found the publication of Cabrera et al. [4] who presents his experience in the use of CVC material in urinary diversion in neonates with hydronephrosis; and that, like us, describes the difficulty in obtaining the nephrostomy catheter set, in addition to not being accessible to patients because of its high cost; probably in Ecuador the situation of accessibility to these procedures is very similar to the one we present in our country, where it is uncommon to have in a general hospital fluoroscopy equipment, tomographs and special materials (nephrostomy set), in addition to the lack of personnel trained in this procedure $[4,12,13]$.

We used to guide the procedure an ultrasound with a convex transducer, which allowed us to observe and control in real time the advance and access of the needle to the renal pelvis; having as an advantage to make this technique an effective, reproducible, economical, fast and safe procedure. Other important qualities for 
the patient and the operator are the non-exposure to radiation and the ease of moving the ultrasound equipment to where it is necessary and to accommodate the patient's position. The success rates described with the different techniques are superior to $90 \%$, similar to those obtained in our experience, in which only minor complications occurred that were self-limiting or resolved with symptomatic $[1,11]$; in the literature they are described with a variable incidence between 2 and 38\%, having as the most frequent the transient hematuria, pain, fever and complications related to the drainage catheter (obstruction, accidental withdrawal). Major complications such as severe hemorrhage, injury to adjacent structures (perforation of the colon, pneumothorax, etc.) and severe infection are also described, with an incidence between 0 and $8 \%[2,11-13]$.

We consider the patient's adequate choice, with a dilated renal pelvis (moderate-severe hydronephrosis), together with a thin catheter $(7 \mathrm{~F})$ and a relatively short distance between the skin and the renal pelvis, in addition to the ultrasound guide that allows to control the advance and access of the needle, has allowed us to have results without major complications in all the cases that met the inclusion and exclusion criteria for the present work.

\section{Conclusion}

The NPGE using the CVC-7F set material is a low cost alternative, safe, reproducible and without exposure to radiation, in patients with moderate to severe hydronephrosis and with distance between dilated renal skin and pelvis, less than the length of the needle used.

\section{Contributions of the authors}

The main idea and bibliographic review was JAP, the procedures were performed by JAP and JES, the data collection and support in the procedures was done by LJF and BAL. The revision of the manuscript and approval of the final version was carried out by MAL, RDK and JCA.

\section{References}

1. Karim R, Sengupta S, Samanta S, Aich RK, Das U, etal. (2010) Percutaneous nephrostomy by direct puncture technique: An observational study. Indian J Nephrol 20: 84-88.
2. Dagli M, Ramchandani P (2011) Percutaneous Nephrostomy: Technical Aspects and Indications. SeminInterventRadiol 28(4): 424-437.

3. Dyer RB, Regan JD, Kavanagh PV, Khatod EG, Chen MY, et al. (2002) Percutaneous nephrostomy with extensions of the technique: Step by step. Radiographics 22: 503-525.

4. Cabrera M (2012) Innovation in the use of a central vascular access catheter for percutaneous nephrostomy. Can we use it as a simple, safe and inexpensive method? Medical Magazine of Our Hospitals 18(2): 8286.

5. Dienstmann R, Da Silva C, Pereira MT, Small IA, Ferreira CG (2008) Palliative percutaneous nephrostomy in recurrent cervical cancer: a retrospective analysis of 50 consecutive cases. J Pain Symptom Manage 36(2): 185-190.

6. (1990) American College of Radiology; Society of Interventional Radiology; Society for Pediatric Radiology, USA.

7. Bloom DA, Morgan RJ and Scardino PL (1989) Thomas Hillier and percutaneous nephrostomy. Urology 33(4): 346-350.

8. Goodwin WE, Casey WC, Woolf W (1955) Percutaneous trocar (needle) nephrostomy in hydronephrosis. J Am Med Assoc 157: 891-894.

9. Pedersen JF, Cowan DF, Kristensen JK, Holm HH, Hancke S, et al. (1976) Ultrasonically-guided percutaneous nephrostomy. Report of 24 cases, Radiology 119(2): 429-431.

10. Pabonn Ramos W, Dariushnia S, Walker T, dOthee B, Ganguli S, et al. (2016) Quality Improvement Guidelines for Percutaneous Nephrostomy. J VascIntervRadiol 27(3): 410-414.

11. Dietrich CF, Lorentzen T, Appelbaum L, Buscarini E, Cantisani V, et al. (2016) EFSUMB guidelines on interventional ultrasound (INVUS), part III - Abdominal treatment procedures (Long Version) Ultraschall Med 37: $27-45$.

12. Wah T, Weston M, Irving H (2004) Percutaneous nephrostomy insertion: outcome data from a prospective multi-operator study at a UK training center. Clinical Radiology 59: 255-261.

13. Skolarikos A, Alivizatos G, Papatsoris A, Constantinides K, Zerbas A, et al. (2006) Ultrasound guided percutaneous nephrostomy performed by urologists: 10 year experience. Urology 68: 495-499.

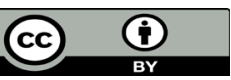

This work is licensed under Creative Commons Attribution 4.0 License

To Submit Your Article Click Here: Submit Article

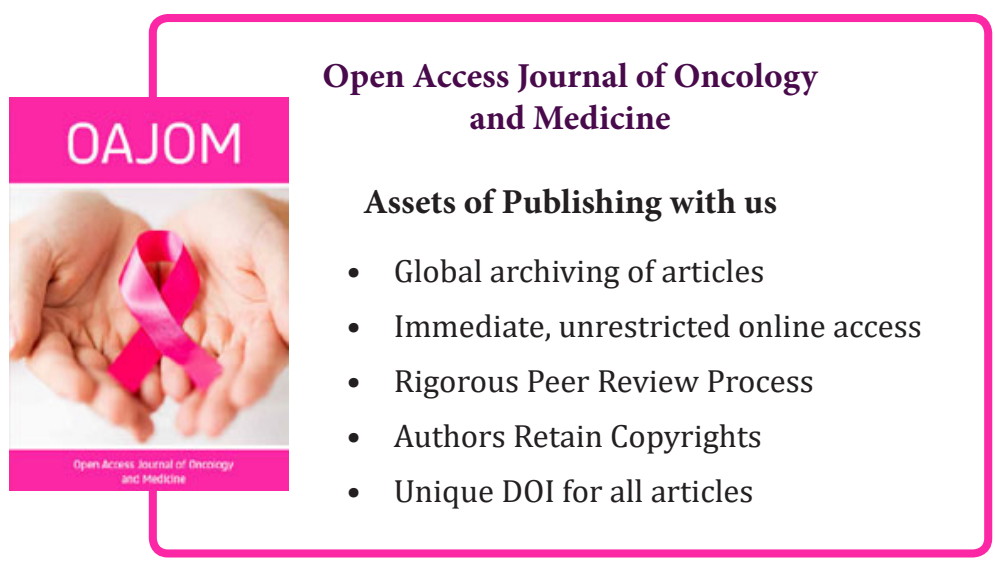

